\title{
Auxin: a molecular trigger of seed development
}

\author{
Duarte D. Figueiredo and Claudia Köhler \\ Department of Plant Biology, Uppsala BioCenter, Swedish University of Agricultural Sciences and Linnean Center \\ for Plant Biology, Uppsala SE-750 07, Sweden
}

The evolution of seeds defines a remarkable landmark in the history of land plants. A developing seed contains three genetically distinct structures: the embryo, the nourishing tissue, and the seed coat. While fertilization is necessary to initiate seed development in most plant species, apomicts have evolved mechanisms allowing seed formation independently of fertilization. Despite their socio-economical relevance, the molecular mechanisms driving seed development have only recently begun to be understood. Here we review the current knowledge on the role of the hormone auxin for the initial development of the three seed structures and as a trigger of fertilization-independent seed development.

Seed development in flowering plants (angiosperms) is preceded by the germination of the pollen grains on the stigma of the flower pistil. The elongating pollen tube carries the two paternal gametes (the sperm cells) and grows through the maternal tissues until it reaches the ovules (Borg et al. 2009). Located within the ovules is the female gametophyte that contains two gametes (the haploid egg cell and the homodiploid central cell) and several accessory cells (two synergids and a species-dependent number of antipodals) (Drews and Koltunow 2011). The female gametophyte (embryo sac) is enclosed within several cell layers of maternal tissues formed by the integuments (Schneitz et al. 1995). When the pollen tube reaches the ovule, it enters through the micropylar integument opening and bursts, releasing the sperm cells (Dresselhaus et al. 2016).

The development of the seed initiates when the two paternal gametes fuse with their maternal counterparts, originating two fertilization products: the diploid embryo and the triploid endosperm. The growth of the embryo, which comprises the next generation, is supported by the mother through the deposition of nutrients in the developing endosperm (Lafon-Placette and Kohler 2014). In most dicot angiosperms, such as Arabidopsis, the embryo consumes most of the endosperm as it develops, and the

[Keywords: auxin; seed; endosperm; seed coat; apomixis; Polycomb group] Corresponding author: claudia.kohler@slu.se

Article is online at http://www.genesdev.org/cgi/doi/10.1101/gad.312546. 118. mature seed contains only a single layer of endosperm cells surrounding the embryo. In contrast, in monocots such as rice and maize, the endosperm is persistent and is consumed only during and after germination by the developing seedling (Yan et al. 2014). Surrounding the two fertilization products is the seed coat, which ensures their protection throughout seed development and after shedding of the seeds from the maternal plant. The seed coat originates from the ovule integuments and is purely of maternal origin (Schneitz et al. 1995; Kelley and Gasser 2009). Importantly, although the integuments do not take part in the fertilization process, their development is triggered after fertilization and accompanies embryo and endosperm growth (Ingram 2010; Figueiredo and Köhler 2014). Thus, the development of a seed is synonymous with the codevelopment of three distinct structures-the embryo, the endosperm, and the seed coat-that, although genetically dissimilar, have to coordinate their growth in order to achieve seed viability and successful transmission of the parental genomes (Ingram 2010; Figueiredo and Köhler 2016). In most plant species, gamete fusion is a strict requirement for seed development to initiate; however, there are exceptions to this rule. Apomixis, a trait present in many angiosperm families, allows initiation of the process autonomously, at least in part. Most apomicts can initiate parthenogenic development of the embryo while still requiring fertilization of the central cell in order for the endosperm to develop. Nevertheless, some apomicts can form seeds completely autonomously without fertilization occurring (Koltunow and Grossniklaus 2003; Hand and Koltunow 2014). Although apomixis was described $>100$ years ago (Winkler 1908) and the potential for agricultural applications is widely recognized (Dwivedi et al. 2010), the mechanisms by which apomicts initiate autonomous seed formation remain elusive. However, this is not that surprising given that the processes that initiate sexual seed development have only recently started to be understood.

(C) 2018 Figueiredo and Köhler This article is distributed exclusively by Cold Spring Harbor Laboratory Press for the first six months after the full-issue publication date (see http://genesdev.cshlp.org/site/misc/ terms.xhtml). After six months, it is available under a Creative Commons License (Attribution-NonCommercial 4.0 International), as described at http://creativecommons.org/licenses/by-nc/4.0/. 
Most progress in unraveling the processes initiating seed development has been made by identifying mutants in the sexual model plant Arabidopsis thaliana that mimic apomicts. Those mutants, collectively referred to as fertilization-independent seed (fis) mutants, have been identified based on their ability to initiate endosperm development in the absence of fertilization /Chaudhury et al. 1997; Ohad et al. 1999; Köhler et al. 2003; Guitton et al. 2004; Roszak and Köhler 2011). This signifies that apomictic pathways are present in nonapomictic species but are under repressive control of FIS proteins. Thus, loss of FIS function leads to the derepression of apomictic pathways and enables the development of seeds without fertilization, also called autonomous seeds.

Cloning of the FIS genes revealed that they code for Polycomb group (PcG) proteins, which assemble to form the multimeric Polycomb-repressive complexes (PRCs) (Derkacheva and Hennig 2014; Mozgova et al. 2015). One of these complexes, PRC2, is responsible for the establishment of the repressive mark trimethylation of Lys27 on histone H3 (Derkacheva and Hennig 2014; Mozgova et al. 2015). This epigenetic mark correlates with local compaction of chromatin, affecting gene expression (Shu et al. 2012). Plants have multigene families coding for PRC2 components, which assemble in distinct complexes that act in different tissues or during different stages of the plant life cycle (Tonosaki and Kinoshita 2015). In Arabidopsis, there are three PRC2 complexes: FIS-PRC2 (acting in the central cell of the female gametophyte) and, in the endosperm, EMF-PRC2 and VRN-PRC2 (acting during sporophytic stages of development) (Derkacheva and Hennig 2014). The ability of mutants in FIS-PRC2 to form autonomous seeds indicates that PRC2 activity is required to repress seed developmental pathways before fertilization (Fig. 1A). This epigenetic block needs to be lifted by fertilization in order for the seed to initiate development.

The plant hormone auxin has been prominently associated with numerous aspects of plant development, including seed formation (Aloni et al. 2006; Overvoorde et al. 2010; Locascio et al. 2014; Pattison et al. 2014; Smit and Weijers 2015). In higher plants, the main auxin is indole3 -acetic acid (IAA), which is biosynthesized from the amino acid tryptophan (Trp) via a two-step process that is strongly conserved among higher plants (Mano and Nemoto 2012; Zhao 2014). The first step involves the removal of the amino group from Trp, which is catalyzed by transaminases belonging to the TRYPTOPHAN AMINOTRANSFERASE OF ARABIDOPSIS (TAA/TAR) family, forming indole-3-pyruvate (IPA) (Stepanova et al. 2008). IPA is then decarboxylated by YUCCA (YUC) flavin mono-oxygenase enzymes, forming IAA (Cheng et al. 2007). Although alternative auxin biosynthesis pathways have been described, TAA-YUC-mediated auxin biosynthesis is the major contributor for the IAA pool in higher plants (Mashiguchi et al. 2011). Auxin is by far the most extensively studied plant hormone, and both its transport and signaling mechanisms have been widely explored and reviewed (Weijers and Wagner 2016; Park et al. 2017).

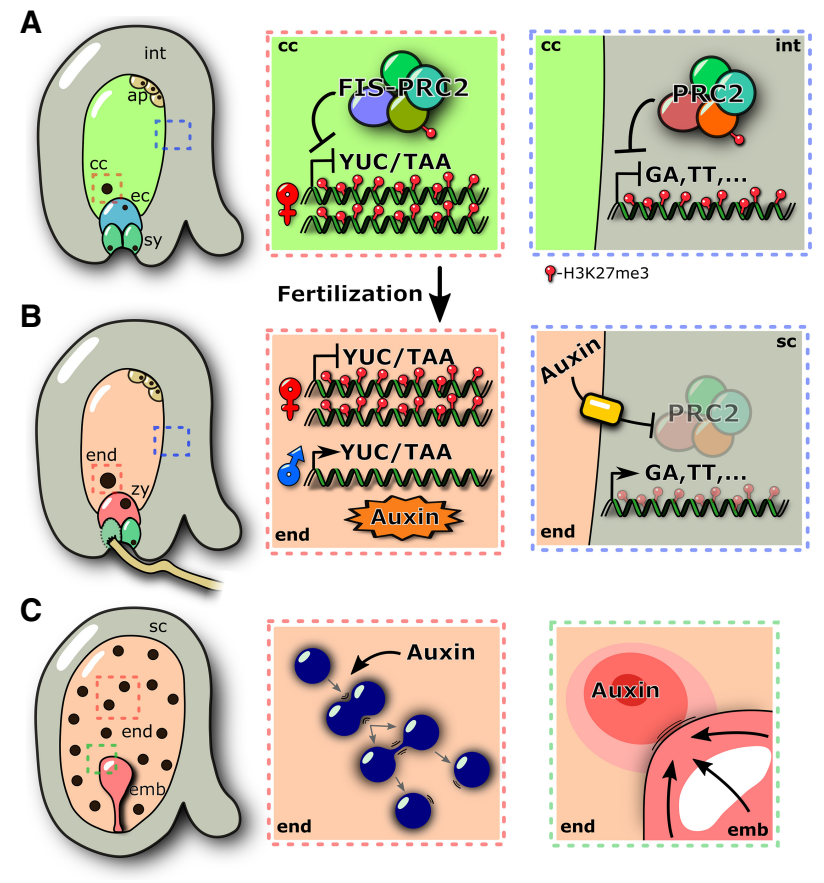

Figure 1. Endosperm-derived auxin regulates seed development. (A) Before fertilization, the expression of auxin biosynthesis genes is repressed in the central cell of the ovule by FIS-PRC2 (red box). Concomitantly, sporophytic PRC2 represses development genes in the integuments (blue box), such as gibberellin (GA) and transparent testa (TT) pathways. (B) Following fertilization, auxin biosynthesis in the endosperm is driven by paternally expressed imprinted genes (red box). Auxin is transported to the maternal tissues, where it removes sporophytic PRC2 function, allowing for the expression of seed coat development genes. $(C)$ In a developing seed, auxin drives the proliferation of the endosperm (red box), and, at least in some species, embryo elongation takes place toward the endosperm auxin maxima (green box). (cc) Central cell; (eg) egg cell; (sy) synergids; (ap) antipodals; (int) integuments; (end) endosperm; (emb) embryo; (sc) seed coat.

It is interesting to note that post-fertilization accumulation of auxin in maize kernels was described $>70$ yr ago (Avery et al. 1942a,b). However, although auxin levels have long been implicated in successful seed development (Rietsema et al. 1953; Teubner 1953; Jennings et al. 1967; Jennings 1971; Torti et al. 1986; Lur and Setter 1993), the molecular mechanisms by which auxin regulates endosperm, embryo, and seed coat development have only recently started to be elucidated (Cheng et al. 2007; Dorcey et al. 2009; Forestan et al. 2010; Bernardi et al. 2012; Figueiredo et al. 2015, 2016; Smit and Weijers 2015). In fact, postfertilization auxin biosynthesis and signaling seem to be the major driving force behind the formation of those three seed structures. In particular, auxin produced in the endosperm has been shown to have a crucial role in modulating the development of the seed coat (Dorcey et al. 2009; Figueiredo et al. 2016). Here we review current knowledge on the role of auxin during the initiation of angiosperm seed development and its potential role as a driver of apomixis. 


\section{Endosperm development is tightly linked to auxin signaling}

The development of the endosperm initiates following the fertilization of the maternal central cell, which points to the requirement of paternal-specific factors for the induction of endosperm proliferation. In fact, sperm entry without karyogamy is sufficient to trigger mitotic divisions of the central cell nucleus; however, sustained endosperm proliferation requires activity of the paternal genome (Aw et al. 2010). The endosperm of flowering plants is characterized by the occurrence of genomic imprinting, an epigenetic phenomenon causing parent-of-origin-specific expression of certain genes (Barlow and Bartolomei 2014; Rodrigues and Zilberman 2015). Imprinted genes can be preferentially or solely expressed from one of the parental alleles and are thereby termed maternally expressed genes (MEGs) or paternally expressed genes (PEGs). Given that endosperm development requires the activity of the paternal genome, PEGs are the prime candidates promoting endosperm proliferation. Genes coding for the auxin biosynthesis enzymes YUC10 and TAR1 are imprinted in the Arabidopsis endosperm (Fig. 1A,B; Gehring et al. 2011; Hsieh et al. 2011; Wolff et al. 2011; Pignatta et al. 2014), raising the possibility that auxin could be the factor driving endosperm development. Indeed, application of auxin to unfertilized ovules or ectopic production of auxin in the central cell is sufficient to trigger its replication and initiate endosperm development (Figueiredo et al. 2015).

The question thus arises of whether the role of auxin as a post-fertilization trigger of endosperm development is conserved in other angiosperms. If so, it is expected that imprinted paternal-specific expression of genes involved in auxin biosynthesis is a conserved feature in angiosperms. Interestingly, there is evidence supporting this notion. In Arabidopsis lyrata, a close relative of $A$. thaliana, the auxin biosynthesis genes YUC10 and TAA1 are imprinted in the endosperm and paternally expressed (Klosinska et al. 2016). Strikingly, orthologs of the same genes, YUC10 and TAA1, are also PEGs in the Capsella genus, which diverged from Arabidopsis $>10$ million years ago (Hatorangan et al. 2016). More notable, however, are the observations that auxin biosynthesis genes are also paternally expressed in the endosperm of monocot species that branched off from dicots $\sim 150$ million years ago (Chaw et al. 2004). Thus, OsYUCCA11, a rice homolog of the Arabidopsis YUC10 gene, is an endosperm-specific PEG in rice seeds (Luo et al. 2011; Du et al. 2014), and the same is true for ZmYUC1 in maize (Zhang et al. 2011; Waters et al. 2013; Xin et al. 2013). The fact that genes involved in auxin biosynthesis are commonly imprinted in the endosperm of distantly related species of angiosperms is quite remarkable because imprinting seems to have little conservation even among closely related species (Waters et al. 2013; Hatorangan et al. 2016). This supports the notion that auxin production following fertilization may be an evolutionarily conserved mechanism that drives endosperm development in flowering plants. In strawberries, homologs of the Arabidopsis YUC10 and TAR1 genes are also specifically expressed in the endo- sperm, albeit whether these genes are imprinted is yet to be determined (Kang et al. 2013). However, it is interesting to note that in the tomato, ToFZY6, the close homolog of YUC10 and YUC11, albeit strongly expressed in seeds (Expósito-Rodríguez et al. 2011), is not described as being imprinted (Florez-Rueda et al. 2016). Given than tomato seeds undergo ab initio cellular endosperm development as opposed to the syncytial proliferation observed in most angiosperms (Lester and Kang 1998), this raises the question of whether auxin production is regulated differently in species with distinct modes of endosperm development. Furthermore, this indicates that auxin activity driven by YUC10/11 homologs is conserved in the endosperm of distinct plant species even if it is not necessarily driven by the paternal alleles. Interestingly, the endosperm-specific YUC10/11 proteins form a clade that seems to be specific for angiosperms and thus coincides with the evolutionary appearance of the endosperm (Abu-Zaitoon et al. 2012).

Correlating with the post-fertilization activity of auxin biosynthesis genes, accumulation of auxin and activation of auxin signaling in the endosperm has been demonstrated in a number of plant species. In Arabidopsis, endosperm proliferation coincides with increased auxin signaling, as measured with the auxin sensor R2D2 (Figueiredo et al. 2015; Liao et al. 2015). In maize, auxin activity is detected during early endosperm development using the DR5 auxin reporter (Chen et al. 2014), correlating with accumulation of IAA in maize endosperm and also in rice seeds (Chourey et al. 2010; Abu-Zaitoon et al. 2012).

We therefore conclude that auxin activity is a hallmark of endosperm development and that auxin is sufficient to initiate endosperm development at least in Arabidopsis (Fig. 1C). The next question we discuss here is whether auxin is also necessary for the continued development of the endosperm throughout seed growth. There is indeed evidence that this is the case in both dicots and monocots. In Arabidopsis, the study of mutants impaired in either auxin biosynthesis or signaling demonstrated that downregulation of auxin dramatically affects endosperm proliferation rates (Figueiredo et al. 2015). Related observations have been reported in maize; the maize mutant defective endosperm-B18 (de18), in which endosperm IAA levels are reduced to $<10 \%$ compared with its wild-type counterpart, has severe endosperm defects (Torti et al. 1986; Bernardi et al. 2012). The de18 mutation affects the PEG ZmYUC1 (Bernardi et al. 2012), linking endosperm development to paternally driven auxin biosynthesis. The endosperm of maize de18 mutants is affected in endoreduplication, while that of Arabidopsis auxin mutants shows striking proliferation defects (Bernardi et al. 2012; Figueiredo et al. 2015). Additionally, both the maize mutants miniature1 (mn1-1) and defective kernel18 (dek18) have a severely reduced endosperm mass, which correlates with the down-regulation of the endosperm-specific ZmYUC1 and ZmTAR1 genes and, consequently, low levels of auxin (LeClere et al. 2008, 2010; Bernardi et al. 2016). Auxin has also been reported to play a pivotal role in regulating endosperm development in the woody plant 
Jatropha curcas, and the auxin response factor JcARF19 in particular was shown to promote the expression of genes coding for cell cycle regulators in the endosperm, such as JcCDKA1, JcCYCD2, and JcCYCD5 (Sun et al. 2017). Similarly, in raspberry and blackberry seeds, the development of the endosperm was described as highly sensitive to auxin levels (Jennings et al. 1967; Jennings 1971). These observations support the role of auxin as a determinant factor in the regulation of endosperm development even if its function may vary between plant species.

Despite the prominent role of auxin during endosperm initiation and growth, in most species, not much is known about the dynamics and transport of this hormone during endosperm development. Auxin transporters in maize endosperm were proposed to be linked to the differentiation of endosperm transfer cells (ETCs) (Forestan et al. 2010), which facilitate the transport of substances between the maternal tissues and the endosperm (Thiel 2014). Genes coding for ZmPIN1 auxin transporters are strongly up-regulated in the endosperm following fertilization, but the ZmPIN1 proteins seem to remain cytoplasmic within the first days of syncytial endosperm development, relocalizing to the plasma membrane and endomembrane system only at the start of cellularization (Forestan et al. 2010). When the endosperm is fully cellularized, ZmPIN1 proteins gather toward the chalazal endosperm region, where auxin accumulates, and the transfer cells begin to differentiate (Forestan et al. 2010). Maize de18 mutants show a lack of ZmPIN1 expression in the endosperm, reduced accumulation of auxin in the transfer cells, and defects in their polarization and differentiation (Forestan and Varotto 2012). These defects likely explain the reduced size of de18 kernels because endosperm filling in maize is highly dependent on the activity of transfer cells (Thiel 2014). This implicates a role of auxin in sustaining endosperm filling in monocots by establishing communication pathways between the maternal tissues and the fertilization products.

\section{Auxin defines embryo polarity and patterning}

Embryo development starts with the fertilization of the egg cell by one of the paternal sperm cells, producing the zygote. Through a series of coordinated cell divisions, the zygote develops into a mature embryo that contains the basic body plan of the future plant (de Vries and Weijers 2017). Embryogenesis is initiated by the polarization of the zygote, which divides asymmetrically, forming a small apical cell and a larger basal cell (Lau et al. 2012; de Vries and Weijers 2017). The former gives rise to the embryo proper, and the latter originates the suspensor that connects the embryo to the seed coat and, except for the apical-most cell in eudicots, does not contribute to the next generation (Kawashima and Goldberg 2010). The establishment of the embryonic apical-basal axis is highly dependent on an asymmetric auxin response, which is maintained by cell-specific localized auxin biosynthesis together with the polarized localization of the auxin efflux transporters PIN1 and PIN7 in Arabidopsis (Möller and
Weijers 2009; Robert et al. 2013). Similarly, the activity of the auxin exporter ZmPIN1 is thought to be important for the proper embryo patterning in maize (Forestan et al. 2010; Locascio et al. 2014).

While the initial steps of embryogenesis in animals are controlled by factors inherited from the egg cytoplasm (Lee et al. 2014), in plants, the parental zygotic genomes contribute at least in part to the initial stages of embryo development. In maize and rice, genome activation occurs shortly after fertilization, and, in particular, genes encoding transcriptional regulators are among early activated genes (Anderson et al. 2017; Chen et al. 2017). To what extent both parental genomes contribute during early embryogenesis remains a matter of debate, and different studies come to opposing conclusions. While activation of the paternal genome was found to be delayed in rice zygotes (Anderson et al. 2017), equivalent parental contributions were reported in maize (Meyer and Scholten 2007). The conclusions regarding paternal genome activation also differ among different Arabidopsis studies (Autran et al. 2011; Nodine and Bartel 2012; Del Toro-De León et al. 2014); nevertheless, there is a consensus that there is early zygotic transcriptional activity in plants, clearly differing from the situation in animals. Interestingly, zygote genome activation in maize coincides with a significant up-regulation of a number of auxin-related genes; namely, those involved in auxin biosynthesis and signaling (Chen et al. 2017). This happens as early as $12 \mathrm{~h}$ after pollination, suggesting that auxin plays a major role in the zygote-to-embryo transition in maize (Chen et al. 2017). Surprisingly, auxin activity is not detected during the initial stages of embryo development in maize but is likely required later in development for the establishment of the apical-basal axis (Forestan et al. 2010; Chen et al. 2014). Importantly, auxin levels in the endosperm are thought to be a driving force for embryo patterning in maize. The embryo-surrounding region (ESR) of the maize endosperm has been proposed to act as a hormonal buffer in the early stages of seed development; as the embryo grows and exits the ESR, it becomes exposed to auxin and cytokinin, which are crucial drivers of embryogenesis (Doll et al. 2017). Given that the endosperm adjacent to the adaxial embryo is particularly rich in auxin signaling (Chen et al. 2014), this suggests a role of endosperm-derived auxin in defining embryo patterning in monocots (Fig. 1C). Indeed, auxin signaling from the adaxial endosperm in maize correlates with apical embryo differentiation and outgrowth, meaning that the embryo elongates in the direction of the endosperm auxin maxima (Chen et al. 2014). The embryo-surrounding endosperm in Arabidopsis seeds also shows a strong accumulation of IAA, as determined by immunolocalization (Avsian-Kretchmer et al. 2002); however, whether endosperm-derived auxin determines embryo patterning in species other than maize (namely, dicots) is yet to be tested. Embryo patterning in monocots and dicots is quite distinct, suggesting the existence of distinct mechanisms regulating this process (Chen et al. 2014; Zhao et al. 2017). Interestingly, Arabidopsis yuc1 yuc4 mutant embryos develop normally, but the embryos mutant for yuc1 yuc4 yuc10 yuc11 show 
marked developmental defects (Cheng et al. 2007). Since both YUC10 and YUC11 are expressed in the endosperm (Robert et al. 2013; Figueiredo et al. 2015), it is tempting to hypothesize that lack of endosperm-derived auxin may contribute to the embryo patterning defects observed in those mutants. Strikingly, the auxin importer LAX1 is specifically expressed in the tips of the cotyledons (Robert et al. 2015). Whether this allows the import of auxin from the endosperm remains to be tested. The endogenous signaling mechanisms driving embryogenesis in both monocots and eudicots have been extensively reviewed and are not covered here (Chen et al. 2014; Smit and Weijers 2015; de Vries and Weijers 2017; Zhao et al. 2017).

\section{Seed coat development is driven by auxin originating in the endosperm}

Seed coat development initiates after double fertilization, similar to what happens with the fertilization products. In contrast, however, the seed coat develops from the maternal integuments and receives no direct paternal genome contribution. Thus, its initiation relies on nonautonomous signaling mechanisms that originate in the pollen or the fertilization products (Roszak and Köhler 2011; Figueiredo and Köhler 2016). Seed coat development is tightly linked to the fertilization of the central cell but not of the egg cell, revealing that the developing endosperm signals to the integuments to initiate the formation of the seed coat (Weijers et al. 2003; Roszak and Köhler 2011). Since the endosperm-derived signal that drives seed coat formation is produced only post-fertilization, imprinted paternally expressed genes are ideal candidates underlying this nonautonomous cell signaling mechanism. Moreover, there are no cytoplasmic connections that have been described between the endosperm and the seed coat; therefore, the communication mechanisms between these two structures are restricted to molecules that can cross membranes (Stadler et al. 2005; Ingram 2010). Thus, possible candidates for the seed coat initiation signal are small molecules, such as signaling peptides (Ingram and Gutierrez-Marcos 2015), and molecules that can be actively exported from the endosperm, such as hormones (Figueiredo and Köhler 2016). In line with this, post-fertilization production of auxin in the endosperm has been linked recently to the initiation of seed coat development (Figueiredo et al. 2016). This is supported by observations that exogenous applications of auxin or ectopic production of auxin in the central cells of unfertilized ovules is sufficient to initiate autonomous development of the seed coat in Arabidopsis (Figueiredo et al. 2016). Furthermore, down-regulation of auxin biosynthesis in the endosperm has a significant impact on seed coat development, revealing that endosperm-derived auxin is necessary in order for the seed coat to develop (Figueiredo et al. 2016). Auxin activity can also be detected after fertilization in the chalazal region of the maternal tissues of maize kernels (Forestan et al. 2010; Chen et al. 2014), suggesting that auxin-initiated development of the maternal seed sporophytic tissues is a conserved feature in angiosperms. In maize seeds, the ZmPIN1a auxin transporter is positioned in the maternal sporophytic tissues near the micropyle, facing the endosperm, implicating the maternal tissues as a possible source of auxin to the endosperm (Chen et al. 2014). Thus, it is possible that the post-fertilization transport of auxin is bidirectional and that the direction of this flow varies between distinct seed domains and/or developmental stages.

How auxin is transported from the endosperm to the surrounding maternal tissues remains to be investigated. Arabidopsis mutants for the MADS-box transcription factor AGL62 fail to export auxin from the endosperm to the integuments, thus failing to form a seed coat, which correlates with the lack of expression of the gene coding for the ABCB-type transporter PGP10 (Figueiredo et al. 2016). Although PGP10 so far has not been shown to transport auxin, its close homologs, PGP1 and PGP19, together with PGP4, have been extensively implicated in cellular auxin export (Geisler et al. 2005; Lin and Wang 2005; Santelia et al. 2005; Geisler and Murphy 2006). Therefore, the transport of auxin between the endosperm and the maternal tissues being mediated by PGP-type transporters is an attractive hypothesis.

Seed coat development initiates only after fertilization, indicating that the developmental transition from integument to seed coat is actively blocked in unfertilized ovules and that this block has to be lifted following fertilization. Mutants for sporophytically acting PcG proteins are able to form a seed coat without fertilization (Roszak and Köhler 2011), indicating that sporophytic PRC2s EMF-PRC2 and VRN-PRC2 block the development of the seed coat before fertilization. This epigenetic block needs to be lifted after fertilization to allow the seed coat to form. This is in line with observations that genes coding for sporophytic PRC2 components are strongly repressed following fertilization (Figueiredo et al. 2016). Importantly, a similar down-regulation of PRC2-coding genes is observed in unfertilized ovules following auxin treatments (Figueiredo et al. 2016). Thus, auxin activity in the seed coat can effectively remove PRC2 function in the integuments, correlating with the initiation of seed coat development (Fig. 1B). Nevertheless, how auxin regulates PRC2-coding genes is yet to be understood.

If sporophytic PRC2 removal is required for seed coat initiation, the question arises of which downstream pathways are repressed by PRC2 in the integuments and become activated following fertilization. One of these signaling pathways, gibberellin (GA) signaling, is tightly linked to seed coat development and, furthermore, acts downstream from auxin (Dorcey et al. 2009; Figueiredo et al. 2016). Application of auxin to unfertilized Arabidopsis ovules is sufficient to activate the expression of genes coding for enzymes involved in GA biosynthesis (Dorcey et al. 2009). Importantly, a similar stimulation of GA biosynthesis is observed in mutants depleted in sporophytic PRC2 (Figueiredo et al. 2016), suggesting that auxin activates GA biosynthesis genes by removing PRC2 in the maternal integuments. Similar to the activation of GA biosynthesis, the accumulation of flavonoids in the seed coat, mediated by the TRANSPARENT TESTA (TT) 
pathway, is also downstream from auxin signaling and PRC2 removal. Both exogenous application of auxin and sporophytic PRC2 depletion in unfertilized ovules lead to the production of proanthocyanidins (Roszak and Köhler 2011; Figueiredo et al. 2016), which normally occurs only following fertilization (Debeaujon et al. 2003; Roszak and Köhler 2011; Figueiredo et al. 2016). Interestingly, flavonoids have been implicated in modulating auxin transport via PGP and PIN-type transporters (Bailly et al. 2008; Santelia et al. 2008). This raises the important question of whether the accumulation of these compounds can influence auxin transport dynamics in the developing seed coat, as proposed previously (Doughty et al. 2014).

Development of the seed sporophytic structures has been linked to the fertilization of the central cell; nevertheless, recent data showed that the content of the pollen tube is sufficient to initiate ovule growth in Arabidopsis (Kasahara et al. 2016; Zhong et al. 2017). Mutants for the transcription factors DROP1 and DROP2 do not produce sperm cells and thus form pollen tubes that do not carry gametes but are still targeted to the ovules (Zhang et al. 2017). Interestingly, drop1 drop2 pollen tube entry and burst can initiate ovule growth (Zhong et al. 2017), albeit to a lesser extent than what happens after exogenous application of auxin or fertilization (Figueiredo et al. 2016). The question thus arises as to the nature of the molecule present in the pollen tube that drives ovule growth. Pollen tubes carry high levels of auxin (Aloni et al. 2006), and, in fact, pollen development is strongly dependent on auxin homeostasis (Zerzour et al. 2009; Ding et al. 2012). Therefore, one likely hypothesis is that auxin present in pollen is released into the female gametophyte upon pollen tube burst, which is sufficient to initiate ovule growth. Nevertheless, this hypothesis remains to be tested, and the presence of additional signaling mechanisms that can initiate seed coat development cannot be ruled out. In summary, seed coat development is triggered by a signal delivered by the pollen and sustained by auxin formed in the endosperm that is transported to the surrounding maternal tissues.

\section{Role of seed-derived auxin in fruit development}

Post-fertilization auxin production in seeds has been implicated in driving fruit development in several plant species (Chareonboonsit et al. 1985; Rotino et al. 1997; Mezzetti et al. 2004; Mariotti et al. 2011; Figueiredo et al. 2016), and exogenous auxin application has long been known to drive fruit development without fertilization, termed parthenocarpy (Gustafson 1936; Thompson 1969; Eeuwens and Schwabe 1975; Chareonboonsit et al. 1985; Vivian-Smith and Koltunow 1999; Serrani et al. 2008). The observation that extracts from the endosperm and seed coat are sufficient to initiate fruit development in peas implicated the production of a signal in the developing seed that induces fruit formation (García-Martínez et al. 1991). Indeed, the endosperm and seed coat were proposed to be the main source of auxin and GA to the maternal receptacle in strawberries and the carpels in Arabi- dopsis (Dorcey et al. 2009; Kang et al. 2013). In support of this, the ectopic expression of bacterial auxin biosynthesis genes in the ovules of several plant species leads to parthenocarpic fruit development, bypassing the requirement for fertilization (Rotino et al. 1997; Mezzetti et al. 2004; Figueiredo et al. 2016). The auxin response factor 8 (ARF8) has been directly implicated in the signal transduction mechanisms leading to fruit formation in distinct plant species (Goetz et al. 2006, 2007; Gorguet et al. 2008; Du et al. 2016). In Arabidopsis, ARF8 is expressed in unfertilized ovules and is strongly repressed after fertilization, and, furthermore, the arf8 mutation leads to fruit development independent of fertilization (Goetz et al. 2006). These observations indicate that the post-fertilization auxin activity not only drives the development of the seed coat but is also a conserved mechanism that nonautonomously modulates fruit formation in distinct plant species through pathways negatively regulated by ARF8.

\section{The role of auxin in apomictic seed formation}

Apomixis allows the formation of seeds without fertilization, a trait of high economic potential that may permit one to fully exploit heterosis by preserving superior genotypes (Koltunow and Grossniklaus 2003). Apomicts have evolved mechanisms to bypass sexual pathways; which involves the formation of functional gametophytes without meiosis (apomeiosis), the formation of embryos without fertilization (parthenogenesis), and the formation of a functional endosperm (Fig. 2A,B). The latter can occur completely autonomously in some species but does involve fertilization in most apomicts (Koltunow and Grossniklaus 2003). Elegant genetic manipulations in Arabidopsis and rice have provided proof of concept that synthetic clonal seed production can be achieved (Fig. 2C; Marimuthu et al. 2011; Mieulet et al. 2016). This entails the combination of mutations bypassing meiosis, leading to the generation of balanced unrecombined diploid gametes. In Arabidopsis, seed formation can be initiated in crosses where one of the parents expresses a modified version of the centromeric histone CENH3, whose genome is then eliminated in the zygote (Marimuthu et al. 2011). Thus, the resulting clonal seeds are supported by a uniparental endosperm, resulting in low seed viability (Marimuthu et al. 2011).

Therefore, one major obstacle that remains to be overcome in producing a synthetic clonal seed is the generation of a functional uniparental endosperm that is able to nourish the clonal embryo. As discussed above, the central role of auxin in initiating seed development makes it reasonable to assume that auxin could be of key importance in this process. This idea is furthered by the finding that both the application of auxin to unfertilized ovules and the depletion of gametophytic FIS-PRC2 function lead to a phenocopy of apomictic endosperm development in the nonapomict $A$. thaliana (Chaudhury et al. 1997; Ohad et al. 1999; Köhler et al. 2003; Roszak and Köhler 2011; Figueiredo et al. 2015, 2016). Thus, a link between FIS-PRC2 function, auxin activity, and activation of 


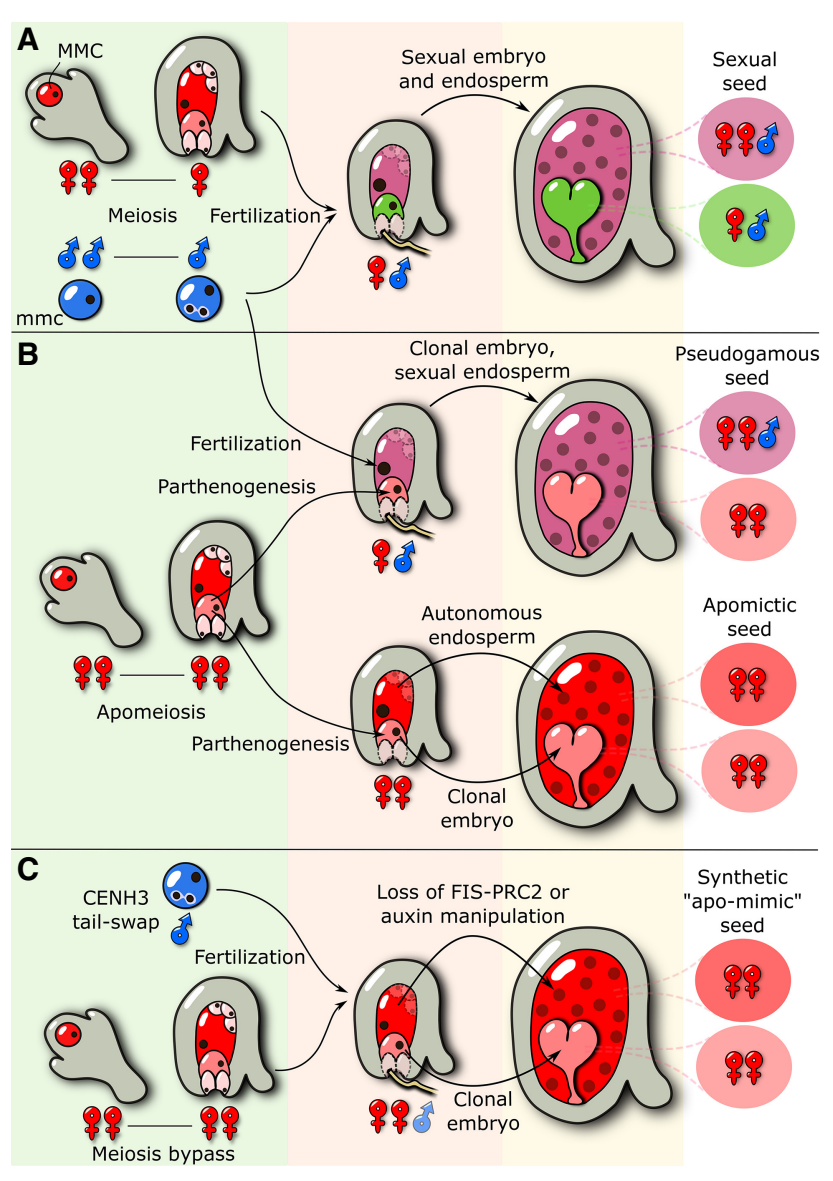

Figure 2. Sexual and asexual modes of seed initiation. $(A)$ In sexual species, meiosis generates haploid gametes that fuse to form a diploid embryo and a triploid endosperm, both containing maternal and paternal genome copies. (MMC) Megaspore mother cell; $(\mathrm{mmc})$ microspore mother cell. $(B)$ In apomictic species undergoing gametophytic apomixis, the maternal plant produces unreduced gametes through apomeiosis and is able to form a parthenogenetic clonal embryo from the egg cell without paternal contribution. Most apomicts are pseudogamous and require fertilization of the central cell, forming a sexual endosperm, while some can initiate autonomous endosperm development, leading to the formation of purely maternally derived seeds. $(C)$ Engineering of apomictic traits in sexual species is possible by combining mutations to allow bypass of meiosis, leading to the formation of unreduced embryo sacs. Expression of modified histone CENH3 in the pollen causes loss of the paternal genome in the zygote, allowing the formation of a clonal embryo. Although the formation of an autonomous endosperm has remained a challenge, loss of FIS-PRC2 function and manipulation of auxin levels are promising strategies for the development of synthetic clonal seeds.

autonomous endosperm development seems plausible. Importantly, FIS-PRC2 is specific to the central cell and its descendent endosperm and contributes to the establishment of genomic imprinting (Mozgova et al. 2015; Rodrigues and Zilberman 2015). In particular, FIS-PRC2 is responsible for the silencing of the maternal alleles of PEGs, ensuring that those genes are not active before fertilization (Moreno-Romero et al. 2016). As discussed above, PEGs (namely, the ones coding for enzymes in- volved in auxin biosynthesis) have been linked to the fertilization-induced initiation of endosperm development (Figueiredo et al. 2015, 2016). This suggests that fis mutants initiate autonomous endosperm development as a consequence of derepressed maternal PEG alleles that ectopically activate seed developmental pathways, bypassing the contribution of the paternal genome. This is in agreement with observations that bypassing genomic imprinting in Arabidopsis allows the development of viable seeds with a purely maternal endosperm (Nowack et al. 2007). Fertilization of wild-type ovules with pollen deficient in cyclin-dependent kinase $A ; 1(C D K A ; 1)$ produces seeds containing embryos but that fail to form a functional endosperm. CDKA;1 codes for a homolog of the budding yeast CDK Cdc $2^{+} / \mathrm{Cdc} 28$, and mutants for this gene produce sperm cells that fail to undergo karyogamy with the female central cell (Mendenhall and Hodge 1998; Aw et al. 2010). Thus, although in some instances sperm cell entry is sufficient to trigger central cell division, the activity of the paternal genome is required for endosperm development (Aw et al. 2010). However, if the $c d k a ; 1 /+$ mutant is used to pollinate a mother plant that lacks FIS-PRC2, this leads to a number of viable, albeit smaller, seeds that contain a purely maternal endosperm (Nowack et al. 2007). The most parsimonious explanation is that genes that are normally repressed by FIS-PRC2 in the central cell, such as the auxin biosynthesis genes, become active and can drive autonomous endosperm proliferation. Thus, the absence of a paternal genome in seeds derived from a fis $\times c d k a ; 1$ cross is compensated for by the activation of maternal PEG alleles in the FIS-PRC2-lacking endosperm.

In line with those predictions, the development of autonomous seeds in fis mutants was shown to coincide with the derepression of the maternal alleles of the PEG YUC10, which is normally expressed only in the endosperm after fertilization (Figueiredo et al. 2015). Concurrently, fis autonomous seeds show ectopic activation of auxin reporters, indicating fertilization-independent activation of auxin signaling (Figueiredo et al. 2015). Furthermore, the observation that exogenous application of auxin or ectopic auxin production in unfertilized ovules leads to a phenocopy of the fis phenotype strongly supports the view that the autonomous seed development in fis mutants is linked to auxin production (Figueiredo et al. 2015). Previously, two quantitative trait loci (QTLs) have been identified to underlie natural variation in autonomous central cell replication of distinct Arabidopsis accessions (Ungru et al. 2008). Importantly, the investigators observed that genes known to influence autonomous seed formation (namely, genes coding for components of FIS-PRC2) were not located in the vicinity of either QTL, implicating the presence of novel unknown regulators of autonomous endosperm development in those genomic regions (Ungru et al. 2008). One of the QTLs on chromosome I that explains $\sim 14 \%$ of the phenotypic variation contains the YUC10 locus, raising the hypothesis that variation in autonomous endosperm formation in different Arabidopsis accessions correlates with differential ectopic expression of auxin biosynthesis genes. 
The link between FIS-PRC2, auxin signaling, and autonomous endosperm development does not seem to be restricted to Arabidopsis but likely extends to other species as well. Subunits of PRC2 are expressed in the endosperm of other plant species, such as maize and rice, suggesting that the prefertilization repression of seed development by PRC2 may be an evolutionarily conserved mechanism (Danilevskaya et al. 2003; Luo et al. 2009; Tonosaki and Kinoshita 2015). Importantly, down-regulation of the rice PRC2 component OsFIE2 leads to autonomous endosperm development, similar to what happens in Arabidopsis (Ohad et al. 1999; Roszak and Köhler 2011; Li et al. 2014). However, whether this autonomous development coincides with the ectopic activation of auxin biosynthesis genes (namely, the imprinted OsYUC11) is yet to be determined. Conversely, in barley, increased expression of the PRC2 components HVFIE and HvE(z) correlates with smaller seed size, consistent with the role of PRC2 in repressing seed development (Kapazoglou et al. 2010; Tonosaki and Kinoshita 2015).

Together, PRC2 suppresses autonomous endosperm development in monocot and dicot species, and auxin biosynthesis genes are imprinted in both groups of angiosperms, making it likely that repression of autonomous endosperm formation by PRC2-mediated control of auxin biosynthesis is a conserved feature in angiosperms. This raises the question of whether similar mechanisms are in place in apomictic species, some of which can develop functional endosperms without paternal contribution. Rather surprisingly, this does not seem to be the case. The genus Hieracium is composed of both sexual species that initiate seed development after gamete fertilization and asexual species that develop seeds independently of fertilization. Quite unexpectedly, down-regulation of the FIS-PRC2 subunit FIE in sexual Hieracium plants does not lead to autonomous proliferation of the central cell (Rodrigues et al. 2008). Furthermore, apomictic Hieracium species require FIE activity for the development of autonomous seeds (Rodrigues et al. 2008). Thus, unlike what happens in nonapomicts such as Arabidopsis or rice, where FIE is a repressor of autonomous seed development (Ohad et al. 1999; Roszak and Köhler 2011; Li et al. 2014), in apomictic Hieracium, FIE seems to promote seed developmental pathways. Consequently, if FIE function is lost in apomictic Hieracium, autonomous seed development is blocked, and the requirement for fertilization is re-established (Rodrigues et al. 2008). Interestingly, FIE function during sporophytic development and even endosperm cellularization in apomictic Hieracium seems to be conserved when compared with its Arabidopsis and rice counterparts (Vinkenoog et al. 2000; Rodrigues et al. 2008; Li et al. 2014). Similarly, HMSI1, the Hieracium ortholog of the FIS-PRC2 component MSI1, does not seem to be involved in autonomous endosperm development in this apomictic species, unlike the case in Arabidopsis (Köhler et al. 2003; Guitton et al. 2004; Rodrigues et al. 2010). These observations suggest that PRC2 function has diverged between apomictic and nonapomictic species regarding the repression of central cell division. Importantly, auxin has been proposed to be involved in the apomictic process in Hieracium (Koltunow et al. 2001; Tucker et al. 2012) and in some pseudogamous species of blackberry that develop parthenogenic embryos but require fertilization to form an endosperm (Jennings et al. 1967). Thus, in the future, it will be interesting to test whether the divergent function of PRC2 correlates with differential regulation of auxin biosynthesis in apomicts, which could be independent of or even positively regulated by FIS-PRC2 function.

As discussed above, the development of the endosperm and seed coat is tightly linked to PRC2 function (Roszak and Köhler 2011; Figueiredo et al. 2015, 2016), in contrast to the initiation of embryo development, which is likely independent of PRC2. Mutations in the PRC2 component MSI1 were reported to promote the transition of the egg cell into a zygote-like structure, which, however, is not sufficient to initiate true embryonic patterning (Guitton and Berger 2005). Nevertheless, since mutations in other PRC2 subunits do not cause the same phenotype /Chaudhury et al. 1997; Ohad et al. 1999) and since MSI1 is part of complexes in addition to PRC2 (Hennig et al. 2005), it seems most likely that embryo initiation is not under the negative control of PRC2 but is controlled by other repressive pathways. This is in line with the predictions that the genetic determinants for parthenogenesis in apomictic species seem to be distinct and independent from the ones regulating autonomous endosperm development (Ogawa et al. 2013; Hand and Koltunow 2014).

\section{Conclusions and perspectives}

The post-fertilization molecular and genetic mechanisms that drive seed development in sexual species are likely to be similar to the mechanisms that allow for fertilizationindependent seed development in apomicts. The central role of auxin in the initiation of seed development makes it reasonable to assume that auxin signaling is underpinning apomictic seed development (Koltunow and Rabiger 2015). However, although auxin is sufficient to initiate autonomous endosperm development in the nonapomict A. thaliana, by inducing the first few divisions of the central cell, auxin alone is not sufficient to form a fully differentiated cellularized endosperm (Figueiredo et al. 2015, 2016). Therefore, additional mechanisms are required in this process that may be under negative epigenetic regulation established by DNA methylation (Vinkenoog et al. 2000). The forthcoming elucidation of those developmental pathways will not only allow the understanding of pressing biological questions but may significantly contribute to crop breeding efforts; namely, by allowing the fixation of heterosis by engineered apomixis (Koltunow and Grossniklaus 2003; Sailer et al. 2016).

\section{Acknowledgments}

This work was funded by a grant from the Swedish Science Foundation (to C.K.) and a grant from the Knut and Alice Wallenberg Foundation (to C.K.). 


\section{References}

Abu-Zaitoon YM, Bennett K, Normanly J, Nonhebel HM. 2012. A large increase in IAA during development of rice grains correlates with the expression of tryptophan aminotransferase OsTAR1 and a grain-specific YUCCA. Physiol Plant 146: 487-499.

Aloni R, Aloni E, Langhans M, Ullrich CI. 2006. Role of auxin in regulating Arabidopsis flower development. Planta 223: 315-328.

Anderson SN, Johnson CS, Chesnut J, Jones DS, Khanday I, Woodhouse M, Li C, Conrad LJ, Russell SD, Sundaresan V. 2017. The zygotic transition is initiated in unicellular plant zygotes with asymmetric activation of parental genomes. Dev Cell 43: 349-358.e344.

Autran D, Baroux C, Raissig MT, Lenormand T, Wittig M, Grob S, Steimer A, Barann M, Klostermeier UC, Leblanc O, et al. 2011. Maternal epigenetic pathways control parental contributions to Arabidopsis early embryogenesis. Cell 145: 707-719.

Avery GS, Berger J, Shalucha B. 1942a. Auxin content of maize kernels during ontogeny, from plants of varying heterotic vigor. Am J Bot 29: 765-772.

Avery GS, Berger J, Shalucha B. 1942b. Auxin storage as related to endosperm type in maize. Bot Gaz 103: 806-808.

Avsian-Kretchmer O, Cheng JC, Chen L, Moctezuma E, Sung ZR. 2002. Indole acetic acid distribution coincides with vascular differentiation pattern during Arabidopsis leaf ontogeny. Plant Physiol 130: 199-209.

Aw SJ, Hamamura Y, Chen Z, Schnittger A, Berger F. 2010. Sperm entry is sufficient to trigger division of the central cell but the paternal genome is required for endosperm development in Arabidopsis. Development 137: 2683-2690.

Bailly A, Sovero V, Vincenzetti V, Santelia D, Bartnik D, Koenig BW, Mancuso S, Martinoia E, Geisler M. 2008. Modulation of P-glycoproteins by auxin transport inhibitors is mediated by interaction with immunophilins. I Biol Chem 283: 2181721826.

Barlow DP, Bartolomei MS. 2014. Genomic imprinting in mammals. Cold Spring Harb Perspect Biol 6: a018382.

Bernardi J, Lanubile A, Li QB, Kumar D, Kladnik A, Cook SD, Ross JJ, Marocco A, Chourey PS. 2012. Impaired auxin biosynthesis in the defective endosperm18 mutant is due to mutational loss of expression in the ZmYuc1 gene encoding endosperm-specific YUCCA1 protein in maize. Plant Physiol 160: $1318-1328$.

Bernardi J, Li Q-B, Gao Y, Zhao Y, Battaglia R, Marocco A, Chourey PS. 2016. The auxin-deficient defective kernel18 (dek18) mutation alters the expression of seed-specific biosynthetic genes in maize. Plant Growth Regul 35: 770-777.

Borg M, Brownfield L, Twell D. 2009. Male gametophyte development: a molecular perspective. J Exp Bot 60: 1465-1478.

Chareonboonsit S, Splittstoesser WE, George WL. 1985. The effects of pollination methods and auxin applications upon parthenocarpic fruit set and development in tomato. Sci Hort 27: $1-8$.

Chaudhury AM, Ming L, Miller C, Craig S, Dennis ES, Peacock WJ. 1997. Fertilization-independent seed development in Arabidopsis thaliana. Proc Natl Acad Sci 94: 4223-4228.

Chaw S-M, Chang C-C, Chen H-L, Li W-H. 2004. Dating the monocot-dicot divergence and the origin of core eudicots using whole chloroplast genomes. J Mol Evol 58: 424-441.

Chen J, Lausser A, Dresselhaus T. 2014. Hormonal responses during early embryogenesis in maize. Biochem Soc Trans 42: 325-331.
Chen J, Strieder N, Krohn NG, Cyprys P, Sprunck S, Engelmann JC, Dresselhaus T. 2017. Zygotic genome activation occurs shortly after fertilization in maize. Plant Cell 29: 2106-2125.

Cheng Y, Dai X, Zhao Y. 2007. Auxin synthesized by the YUCCA flavin monooxygenases is essential for embryogenesis and leaf formation in Arabidopsis. Plant Cell 19: 2430-2439.

Chourey PS, Li QB, Kumar D. 2010. Sugar-hormone cross-talk in seed development: two redundant pathways of IAA biosynthesis are regulated differentially in the invertase-deficient miniature1 (mn1) seed mutant in maize. Mol Plant 3: 1026-1036.

Danilevskaya ON, Hermon P, Hantke S, Muszynski MG, Kollipara K, Ananiev EV. 2003. Duplicated fie genes in maize: expression pattern and imprinting suggest distinct functions. Plant Cell 15: 425-438.

Debeaujon I, Nesi N, Perez P, Devic M, Grandjean O, Caboche M, Lepiniec L. 2003. Proanthocyanidin-accumulating cells in Arabidopsis testa: regulation of differentiation and role in seed development. Plant Cell 15: 2514-2531.

Del Toro-De León G, García-Aguilar M, Gillmor CS. 2014. Nonequivalent contributions of maternal and paternal genomes to early plant embryogenesis. Nature 514: 624.

Derkacheva M, Hennig L. 2014. Variations on a theme: Polycomb group proteins in plants. J Exp Bot 65: 2769-2784.

de Vries SC, Weijers D. 2017. Plant embryogenesis. Curr Biol 27: R870-R873.

Ding Z, Wang B, Moreno I, Duplakova N, Simon S, Carraro N, Reemmer J, Pencik A, Chen X, Tejos R, et al. 2012. ER-localized auxin transporter PIN8 regulates auxin homeostasis and male gametophyte development in Arabidopsis. Nat Commun 3: 941.

Doll NM, Depege-Fargeix N, Rogowsky PM, Widiez T. 2017. Signaling in early maize kernel development. Mol Plant 10: 375-388.

Dorcey E, Urbez C, Blazquez MA, Carbonell J, Perez-Amador MA. 2009. Fertilization-dependent auxin response in ovules triggers fruit development through the modulation of gibberellin metabolism in Arabidopsis. Plant J 58: 318-332.

Doughty J, Aljabri M, Scott RJ. 2014. Flavonoids and the regulation of seed size in Arabidopsis. Biochem Soc Trans 42: 364-369.

Dresselhaus T, Sprunck S, Wessel GM. 2016. Fertilization mechanisms in flowering plants. Curr Biol 26: R125-R139.

Drews GN, Koltunow AM. 2011. The female gametophyte. Arabidopsis Book 9: $\mathrm{e} 0155$.

Du M, Luo M, Zhang R, Finnegan EJ, Koltunow AM. 2014. Imprinting in rice: the role of DNA and histone methylation in modulating parent-of-origin specific expression and determining transcript start sites. Plant J 79: 232-242.

Du L, Bao C, Hu T, Zhu Q, Hu H, He Q, Mao W. 2016. SmARF8, a transcription factor involved in parthenocarpy in eggplant. Mol Genet Genomics 291: 93-105.

Dwivedi SL, Perotti E, Upadhyaya HD, Ortiz R. 2010. Sexual and apomictic plant reproduction in the genomics era: exploring the mechanisms potentially useful in crop plants. Sex Plant Reprod 23: 265-279.

Eeuwens CJ, Schwabe WW. 1975. Seed and pod wall development in Pisum sativum, L. in relation to extracted and applied hormones. J Exp Bot 26: 1-14.

Expósito-Rodríguez M, Borges AA, Borges-Pérez A, Pérez JA. 2011. Gene structure and spatiotemporal expression profile of tomato genes encoding YUCCA-like flavin monooxygenases: the ToFZY gene family. Plant Phys Biochem 49: 782-791.

Figueiredo DD, Köhler C. 2014. Signalling events regulating seed coat development. Biochem Soc Trans 42: 358-363. 
Figueiredo DD, Köhler C. 2016. Bridging the generation gap: communication between maternal sporophyte, female gametophyte and fertilization products. Curr Opin Plant Biol 29: 16-20.

Figueiredo DD, Batista RA, Roszak PJ, Köhler C. 2015. Auxin production couples endosperm development to fertilization. Nat Plants 1: 15184.

Figueiredo DD, Batista RA, Roszak PJ, Hennig L, Kohler C. 2016. Auxin production in the endosperm drives seed coat development in Arabidopsis. Elife 5: e20542.

Florez-Rueda AM, Paris M, Schmidt A, Widmer A, Grossniklaus $\mathrm{U}$, Stadler T. 2016. Genomic imprinting in the endosperm is systematically perturbed in abortive hybrid tomato seeds. Mol Biol Evol 33: 2935-2946.

Forestan C, Varotto S. 2012. The role of PIN auxin efflux carriers in polar auxin transport and accumulation and their effect on shaping maize development. Mol Plant 5: 787-798.

Forestan C, Meda S, Varotto S. 2010. ZmPIN1-mediated auxin transport is related to cellular differentiation during maize embryogenesis and endosperm development. Plant Physiol 152: $1373-1390$.

García-Martínez JL, Martí M, Sabater T, Maldonado A, Vercher Y. 1991. Development of fertilized ovules and their role in the growth of the pea pod. Physiol Plant 83: 411-416.

Gehring M, Missirian V, Henikoff S. 2011. Genomic analysis of parent-of-origin allelic expression in Arabidopsis thaliana seeds. PLoS One 6: e23687.

Geisler M, Murphy AS. 2006. The ABC of auxin transport: the role of p-glycoproteins in plant development. FEBS Lett 580: 1094-1102.

Geisler M, Blakeslee JJ, Bouchard R, Lee OR, Vincenzetti V, Bandyopadhyay A, Titapiwatanakun B, Peer WA, Bailly A, Richards EL, et al. 2005. Cellular efflux of auxin catalyzed by the Arabidopsis MDR/PGP transporter AtPGP1. Plant $J$ 44: 179-194.

Goetz M, Vivian-Smith A, Johnson SD, Koltunow AM. 2006. AUXIN RESPONSE FACTOR8 is a negative regulator of fruit initiation in Arabidopsis. Plant Cell 18: 1873-1886.

Goetz M, Hooper LC, Johnson SD, Rodrigues JCM, Vivian-Smith A, Koltunow AM. 2007. Expression of aberrant forms of $A U X$ IN RESPONSE FACTOR8 stimulates parthenocarpy in Arabidopsis and tomato. Plant Physiol 145: 351-366.

Gorguet B, Eggink PM, Ocaña J, Tiwari A, Schipper D, Finkers R, Visser RGF, van Heusden AW. 2008. Mapping and characterization of novel parthenocarpy QTLs in tomato. Theor Appl Genet 116: 755-767.

Guitton A-E, Berger F. 2005. Loss of function of MULTICOPY SUPPRESSOR OF IRA 1 produces nonviable parthenogenetic embryos in Arabidopsis. Curr Biol 15: 750-754.

Guitton AE, Page DR, Chambrier P, Lionnet C, Faure JE, Grossniklaus U, Berger F. 2004. Identification of new members of fertilisation independent seed Polycomb group pathway involved in the control of seed development in Arabidopsis thaliana. Development 131: 2971-2981.

Gustafson FG. 1936. Inducement of fruit development by growthpromoting chemicals. Proc Natl Acad Sci 22: 628-636.

Hand ML, Koltunow AMG. 2014. The genetic control of apomixis: asexual seed formation. Genetics 197: 441-450.

Hatorangan MR, Laenen B, Steige KA, Slotte T, Kohler C. 2016. Rapid evolution of genomic imprinting in two species of the Brassicaceae. Plant Cell 28: 1815-1827.

Hennig L, Bouveret R, Gruissem W. 2005. MSI1-like proteins: an escort service for chromatin assembly and remodeling complexes. Trends Cell Biol 15: 295-302.
Hsieh TF, Shin J, Uzawa R, Silva P, Cohen S, Bauer MJ, Hashimoto M, Kirkbride RC, Harada JJ, Zilberman D, et al. 2011. Regulation of imprinted gene expression in Arabidopsis endosperm. Proc Natl Acad Sci 108: 1755-1762.

Ingram GC. 2010. Family life at close quarters: communication and constraint in angiosperm seed development. Protoplasma 247: 195-214.

Ingram G, Gutierrez-Marcos J. 2015. Peptide signalling during angiosperm seed development. J Exp Bot 66: 5151-5159.

Jennings DL. 1971. Some genetic factors affecting the development of the endocarp, endosperm and embryo in raspberries. New Phytol 70: 885-895.

Jennings DL, Craig DL, Topham PB. 1967. The role of the male parent in the reproduction of Rubus. Heredity 22: 43.

Kang C, Darwish O, Geretz A, Shahan R, Alkharouf N, Liu Z. 2013. Genome-scale transcriptomic insights into early-stage fruit development in woodland strawberry Fragaria vesca. Plant Cell 25: 1960-1978.

Kapazoglou A, Tondelli A, Papaefthimiou D, Ampatzidou H, Francia E, Stanca MA, Bladenopoulos K, Tsaftaris AS. 2010. Epigenetic chromatin modifiers in barley: IV. The study of barley Polycomb group (PcG) genes during seed development and in response to external ABA. BMC Plant Biol 10: 73.

Kasahara RD, Notaguchi M, Nagahara S, Suzuki T, Susaki D, Honma Y, Maruyama D, Higashiyama T. 2016. Pollen tube contents initiate ovule enlargement and enhance seed coat development without fertilization. Sci Adv 2: e1600554.

Kawashima T, Goldberg RB. 2010. The suspensor: not just suspending the embryo. Trends Plant Sci 15: 23-30.

Kelley DR, Gasser CS. 2009. Ovule development: genetic trends and evolutionary considerations. Sex Plant Reprod 22: 229-234.

Klosinska M, Picard CL, Gehring M. 2016. Conserved imprinting associated with unique epigenetic signatures in the Arabidopsis genus. Nat Plants 2: 16145.

Köhler C, Hennig L, Bouveret R, Gheyselinck J, Grossniklaus U, Gruissem W. 2003. Arabidopsis MSI1 is a component of the MEA/FIE Polycomb group complex and required for seed development. EMBO J 22: 4804-4814.

Koltunow AM, Grossniklaus U. 2003. Apomixis: a developmental perspective. Annu Rev Plant Biol 54: 547-574.

Koltunow A, Rabiger DS. 2015. Development: turning on endosperm in seeds. Nat Plants 1: 15189.

Koltunow AM, Johnson SD, Lynch M, Yoshihara T, Costantino P. 2001. Expression of rolB in apomictic Hieracium piloselloides Vill. causes ectopic meristems in planta and changes in ovule formation, where apomixis initiates at higher frequency. Planta 214: 196-205.

Lafon-Placette C, Kohler C. 2014. Embryo and endosperm, partners in seed development. Curr Opin Plant Biol 17: 64-69.

Lau S, Slane D, Herud O, Kong J, Jürgens G. 2012. Early embryogenesis in flowering plants: setting up the basic body pattern. Annu Rev Plant Biol 63: 483-506.

LeClere S, Schmelz EA, Chourey PS. 2008. Cell wall invertase-deficient miniature1 kernels have altered phytohormone levels. Phytochemistry 69: 692-699.

LeClere S, Schmelz EA, Chourey PS. 2010. Sugar levels regulate tryptophan-dependent auxin biosynthesis in developing maize kernels. Plant Physiol 153: 306-318.

Lee MT, Bonneau AR, Giraldez AJ. 2014. Zygotic genome activation during the maternal-to-zygotic transition. Ann Rev Cell Dev Biol 30: 581-613.

Lester RN, Kang JH. 1998. Embryo and endosperm function and failure in Solanum species and hybrids. Ann Bot 82: 445-453. 
Li S, Zhou B, Peng X, Kuang Q, Huang X, Yao J, Du B, Sun MX. 2014. OsFIE2 plays an essential role in the regulation of rice vegetative and reproductive development. New Phytol 201: 66-79.

Liao CY, Smet W, Brunoud G, Yoshida S, Vernoux T, Weijers D. 2015. Reporters for sensitive and quantitative measurement of auxin response. Nat Methods 12: 207-210.

Lin R, Wang H. 2005. Two homologous ATP-binding cassette transporter proteins, AtMDR1 and AtPGP1, regulate Arabidopsis photomorphogenesis and root development by mediating polar auxin transport. Plant Physiol 138: 949-964.

Locascio A, Roig-Villanova I, Bernardi J, Varotto S. 2014. Current perspectives on the hormonal control of seed development in Arabidopsis and maize: a focus on auxin. Front Plant Sci 5: 412.

Luo M, Platten D, Chaudhury A, Peacock WJ, Dennis ES. 2009. Expression, imprinting, and evolution of rice homologs of the Polycomb Group genes. Mol Plant 2: 711-723.

Luo M, Taylor JM, Spriggs A, Zhang H, Wu X, Russell S, Singh M, Koltunow A. 2011. A genome-wide survey of imprinted genes in rice seeds reveals imprinting primarily occurs in the endosperm. PLoS Genet 7: e1002125.

Lur HS, Setter TL. 1993. Role of auxin in maize endosperm development (timing of nuclear DNA endoreduplication, zein expression, and cytokinin). Plant Physiol 103: 273-280.

Mano Y, Nemoto K. 2012. The pathway of auxin biosynthesis in plants. J Exp Bot 63: 2853-2872.

Marimuthu MPA, Jolivet S, Ravi M, Pereira L, Davda JN, Cromer L, Wang L, Nogué F, Chan SWL, Siddiqi I, et al. 2011. Synthetic clonal reproduction through seeds. Science 331: 876-876.

Mariotti L, Picciarelli P, Lombardi L, Ceccarelli N. 2011. Fruit-set and early fruit growth in tomato are associated with increases in indoleacetic acid, cytokinin, and bioactive gibberellin contents. J Plant Growth Regul 30: 405.

Mashiguchi K, Tanaka K, Sakai T, Sugawara S, Kawaide H, Natsume M, Hanada A, Yaeno T, Shirasu K, Yao H, et al. 2011. The main auxin biosynthesis pathway in Arabidopsis. Proc Nat1 Acad Sci 108: 18512-18517.

Mendenhall MD, Hodge AE. 1998. Regulation of Cdc28 cyclindependent protein kinase activity during the cell cycle of the yeast Saccharomyces cerevisiae. Microbiol Mol Biol Rev 62: 1191-1243.

Meyer S, Scholten S. 2007. Equivalent parental contribution to early plant zygotic development. Curr Biol 17: 1686-1691.

Mezzetti B, Landi L, Pandolfini T, Spena A. 2004. The defH9iaaM auxin-synthesizing gene increases plant fecundity and fruit production in strawberry and raspberry. BMC Biotechnol 4: 4.

Mieulet D, Jolivet S, Rivard M, Cromer L, Vernet A, Mayonove P, Pereira L, Droc G, Courtois B, Guiderdoni E, et al. 2016. Turning rice meiosis into mitosis. Cell Res 26: 1242.

Möller B, Weijers D. 2009. Auxin control of embryo patterning. Cold Spring Harb Perspect Biol 1: a001545.

Moreno-Romero J, Jiang H, Santos-González J, Köhler C. 2016. Parental epigenetic asymmetry of PRC2-mediated histone modifications in the Arabidopsis endosperm. EMBO I 35: 1298-1311.

Mozgova I, Kohler C, Hennig L. 2015. Keeping the gate closed: functions of the polycomb repressive complex PRC2 in development. Plant J 83: 121-132.

Nodine MD, Bartel DP. 2012. Maternal and paternal genomes contribute equally to the transcriptome of early plant embryos. Nature 482: 94-97.
Nowack MK, Shirzadi R, Dissmeyer N, Dolf A, Endl E, Grini PE, Schnittger A. 2007. Bypassing genomic imprinting allows seed development. Nature 447: 312-315.

Ogawa D, Johnson SD, Henderson ST, Koltunow AMG. 2013. Genetic separation of autonomous endosperm formation (AutE) from the two other components of apomixis in Hieracium. Plant Reprod 26: 113-123.

Ohad N, Yadegari R, Margossian L, Hannon M, Michaeli D, Harada JJ, Goldberg RB, Fischer RL. 1999. Mutations in FIE, a WD polycomb group gene, allow endosperm development without fertilization. Plant Cell 11: 407-415.

Overvoorde P, Fukaki H, Beeckman T. 2010. Auxin control of root development. Cold Spring Harb Perspect Biol 2: a001537.

Park J, Lee Y, Martinoia E, Geisler M. 2017. Plant hormone transporters: what we know and what we would like to know. BMC Biol 15: 93.

Pattison RJ, Csukasi F, Catalá C. 2014. Mechanisms regulating auxin action during fruit development. Physiol Plant 151: 62-72.

Pignatta D, Erdmann RM, Scheer E, Picard CL, Bell GW, Gehring M. 2014. Natural epigenetic polymorphisms lead to intraspecific variation in Arabidopsis gene imprinting. eLife 3: e03198.

Rietsema J, Satina S, Blakeslee AF. 1953. The effect of indole-3acetic acid on datura embryos. Proc Natl Acad Sci 39: 924-933.

Robert HS, Grones P, Stepanova AN, Robles LM, Lokerse AS, Alonso JM, Weijers D, Friml J. 2013. Local auxin sources orient the apical-basal axis in Arabidopsis embryos. Curr Biol 23: 2506-2512.

Robert HS, Grunewald W, Sauer M, Cannoot B, Soriano M, Swarup R, Weijers D, Bennett M, Boutilier K, Friml J. 2015. Plant embryogenesis requires AUX/LAX-mediated auxin influx. Development 142: 702-711.

Rodrigues JA, Zilberman D. 2015. Evolution and function of genomic imprinting in plants. Genes Dev 29: 2517-2531.

Rodrigues JC, Tucker MR, Johnson SD, Hrmova M, Koltunow AM. 2008. Sexual and apomictic seed formation in Hieracium requires the plant polycomb-group gene FERTILIZATION INDEPENDENT ENDOSPERM. Plant Cell 20: 2372-2386.

Rodrigues JCM, Okada T, Johnson SD, Koltunow AM. 2010. A MULTICOPY SUPPRESSOR OF IRA1 (MSI1) homologue is not associated with the switch to autonomous seed development in apomictic (asexual) Hieracium plants. Plant Science 179: 590-597.

Roszak P, Köhler C. 2011. Polycomb group proteins are required to couple seed coat initiation to fertilization. Proc Nat1 Acad Sci 108: 20826-20831.

Rotino GL, Perri E, Zottini M, Sommer H, Spena A. 1997. Genetic engineering of parthenocarpic plants. Nat Biotechnol 15: 1398.

Sailer C, Schmid B, Grossniklaus U. 2016. Apomixis allows the transgenerational fixation of phenotypes in hybrid plants. Curr Biol 26: 331-337.

Santelia D, Vincenzetti V, Azzarello E, Bovet L, Fukao Y, Duchtig P, Mancuso S, Martinoia E, Geisler M. 2005. MDR-like ABC transporter AtPGP4 is involved in auxin-mediated lateral root and root hair development. FEBS Lett 579: 5399-5406.

Santelia D, Henrichs S, Vincenzetti V, Sauer M, Bigler L, Klein M, Bailly A, Lee Y, Friml J, Geisler M, et al. 2008. Flavonoids redirect PIN-mediated polar auxin fluxes during root gravitropic responses. J Biol Chem 283: 31218-31226.

Schneitz K, Hülskamp M, Pruitt RE. 1995. Wild-type ovule development in Arabidopsis thaliana: a light microscope study of cleared whole-mount tissue. Plant J 7: 731-749. 
Serrani JC, Ruiz-Rivero O, Fos M, García-Martínez JL. 2008. Auxin-induced fruit-set in tomato is mediated in part by gibberellins. Plant J 56: 922-934.

Shu H, Wildhaber T, Siretskiy A, Gruissem W, Hennig L. 2012. Distinct modes of DNA accessibility in plant chromatin. Nat Commun 3: 1281.

Smit ME, Weijers D. 2015. The role of auxin signaling in early embryo pattern formation. Curr Opin Plant Biol 28: 99-105.

Stadler R, Lauterbach C, Sauer N. 2005. Cell-to-cell movement of green fluorescent protein reveals post-phloem transport in the outer integument and identifies symplastic domains in Arabidopsis seeds and embryos. Plant Physiol 139: 701-712.

Stepanova AN, Robertson-Hoyt J, Yun J, Benavente LM, Xie DY, Dolezal K, Schlereth A, Jurgens G, Alonso JM. 2008. TAA1mediated auxin biosynthesis is essential for hormone crosstalk and plant development. Cell 133: 177-191.

Sun Y, Wang C, Wang N, Jiang X, Mao H, Zhu C, Wen F, Wang X, Lu Z, Yue G, et al. 2017. Manipulation of Auxin response factor 19 affects seed size in the woody perennial Jatropha curcas. Sci Rep 7: 40844.

Teubner FG. 1953. Identification of the auxin present in apple endosperm. Science 118: 418-418.

Thiel J. 2014. Development of endosperm transfer cells in barley. Front Plant Sci 5: 108.

Thompson PA. 1969. The effect of applied growth substances on development of the strawberry fruit: II. Interactions of auxins and gibberellins. J Exp Bot 20: 629-647.

Tonosaki K, Kinoshita T. 2015. Possible roles for polycomb repressive complex 2 in cereal endosperm. Front Plant Sci 6: 144.

Torti G, Manzocchi L, Salamini F. 1986. Free and bound indoleacetic acid is low in the endosperm of the maize mutant defective-endosperm-B18. Theor Appl Genet 72: 602-605.

Tucker MR, Okada T, Johnson SD, Takaiwa F, Koltunow AM. 2012. Sporophytic ovule tissues modulate the initiation and progression of apomixis in Hieracium. I Exp Bot 63: 32293241.

Ungru A, Nowack MK, Reymond M, Shirzadi R, Kumar M, Biewers S, Grini PE, Schnittger A. 2008. Natural variation in the degree of autonomous endosperm formation reveals independence and constraints of embryo growth during seed development in Arabidopsis thaliana. Genetics 179: 829-841.

Vinkenoog R, Spielman M, Adams S, Fischer RL, Dickinson HG, Scott RJ. 2000. Hypomethylation promotes autonomous endosperm development and rescues postfertilization lethality in fie mutants. Plant Cell 12: 2271-2282.
Vivian-Smith A, Koltunow AM. 1999. Genetic analysis of growthregulator-induced parthenocarpy in Arabidopsis. Plant Physiol 121: 437-452.

Waters AJ, Bilinski P, Eichten SR, Vaughn MW, Ross-Ibarra J, Gehring M, Springer NM. 2013. Comprehensive analysis of imprinted genes in maize reveals allelic variation for imprinting and limited conservation with other species. Proc Nat1 Acad Sci 110: 19639-19644.

Weijers D, Wagner D. 2016. Transcriptional responses to the auxin hormone. Annu Rev Plant Biol 67: 539-574.

Weijers D, Van Hamburg JP, Van Rijn E, Hooykaas PJ, Offringa R. 2003. Diphtheria toxin-mediated cell ablation reveals interregional communication during Arabidopsis seed development. Plant Physiol 133: 1882-1892.

Winkler H. 1908. Über parthenogenesis und apogamie im pflanzenreich. Progressus Rei Bot 2: 293-454.

Wolff P, Weinhofer I, Seguin J, Roszak P, Beisel C, Donoghue MT, Spillane C, Nordborg M, Rehmsmeier M, Köhler C. 2011. High-resolution analysis of parent-of-origin allelic expression in the Arabidopsis endosperm. PLoS Genet 7: e1002126.

Xin M, Yang R, Li G, Chen H, Laurie J, Ma C, Wang D, Yao Y, Larkins BA, Sun Q, et al. 2013. Dynamic expression of imprinted genes associates with maternally controlled nutrient allocation during maize endosperm development. Plant Cell 25: 3212-3227.

Yan D, Duermeyer L, Leoveanu C, Nambara E. 2014. The functions of the endosperm during seed germination. Plant Cell Physiol 55: 1521-1533.

Zerzour R, Kroeger J, Geitmann A. 2009. Polar growth in pollen tubes is associated with spatially confined dynamic changes in cell mechanical properties. Dev Biol 334: 437-446.

Zhang M, Zhao H, Xie S, Chen J, Xu Y, Wang K, Zhao H, Guan H, $\mathrm{Hu} \mathrm{X}$, Jiao Y, et al. 2011. Extensive, clustered parental imprinting of protein-coding and noncoding RNAs in developing maize endosperm. Proc Natl Acad Sci 108: 20042-20047.

Zhang J, Huang Q, Zhong S, Bleckmann A, Huang J, Guo X, Lin Q, Gu H, Dong J, Dresselhaus T, et al. 2017. Sperm cells are passive cargo of the pollen tube in plant fertilization. Nat Plants 3: 17079.

Zhao Y. 2014. Auxin biosynthesis. Arabidopsis Book 12: e0173.

Zhao P, Begcy K, Dresselhaus T, Sun M-X. 2017. Does early embryogenesis in eudicots and monocots involve the same mechanism and molecular players? Plant Physiol 173: 130142.

Zhong S, Zhang J, Qu L-J. 2017. The signals to trigger the initiation of ovule enlargement are from the pollen tubes: the direct evidence. J Integr Plant Biol 59: 600-603. 


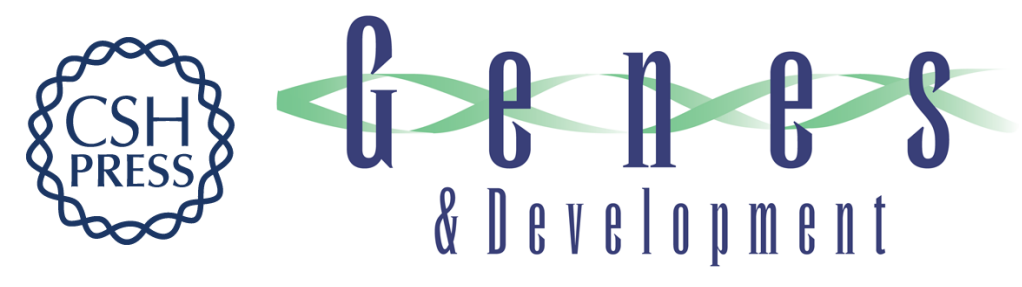

\section{Auxin: a molecular trigger of seed development}

Duarte D. Figueiredo and Claudia Köhler

Genes Dev. 2018, 32:

Access the most recent version at doi:10.1101/gad.312546.118

References This article cites 143 articles, 50 of which can be accessed free at: http://genesdev.cshlp.org/content/32/7-8/479.full.html\#ref-list-1

Creative This article is distributed exclusively by Cold Spring Harbor Laboratory Press for the first Commons six months after the full-issue publication date (see License http://genesdev.cshlp.org/site/misc/terms.xhtml). After six months, it is available under a Creative Commons License (Attribution-NonCommercial 4.0 International), as described at http://creativecommons.org/licenses/by-nc/4.0/.

Email Alerting Receive free email alerts when new articles cite this article - sign up in the box at the top Service right corner of the article or click here.

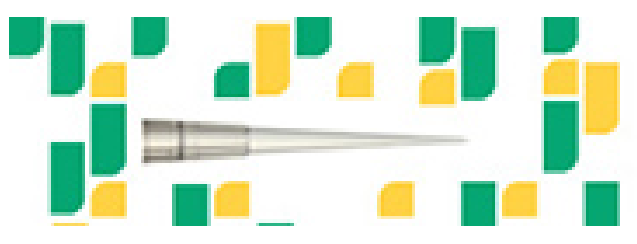

Focused on your science. 